\title{
Carnets
}

Revue électronique d'études françaises de l'APEF

Deuxième série - 8 | 2016

Du Français en cause aux causes du français

\section{Images associées à l'usage du français en milieu professionnel marocain : Cas des PME}

\section{Toufik Majdi}

\section{(2) OpenEdition}

Journals

\section{Édition électronique}

URL : http://journals.openedition.org/carnets/1940

DOI : 10.4000/carnets. 1940

ISSN : 1646-7698

Éditeur

APEF

\section{Référence électronique}

Toufik Majdi, « Images associées à l'usage du français en milieu professionnel marocain : Cas des PME », Carnets [En ligne], Deuxième série - 8 | 2016, mis en ligne le 30 novembre 2016, consulté le 19 avril 2019. URL : http://journals.openedition.org/carnets/1940 ; DOI : 10.4000/carnets.1940

Ce document a été généré automatiquement le 19 avril 2019.

\section{(c) (i) (8)}

Carnets est mis à disposition selon les termes de la licence Creative Commons - Atribution - Pas d'utilisation commerciale 4.0 International. 


\title{
Images associées à l'usage du français en milieu professionnel marocain : Cas des PME
}

\author{
Toufik Majdi
}

\section{Introduction}

1 La langue française s'est enracinée au Maroc depuis l'ère du colon. Mais à l'heure actuelle, la place qu'elle occupe dans le concert des langues en présence la dote d'un rôle instrumental, voire culturel et fait d'elle une langue de modernité, de progrès et d'ouverture sur l'occident. D'ailleurs, dans l'imaginaire de l'esprit marocain, la langue française serait associée à des images valorisantes ayant trait à la réussite, à l'intelligence, au pouvoir, au prestige et à toutes les valeurs superlatives qui en découlent.

2 Ceci dit, il ne faut pas oublier que le Marocain apprend et pratique le français en « homme libre » et sans risque d'aliénation. Dans ce cas, le français serait considéré comme une richesse supplémentaire qui vient appuyer le courant de base qu'est l'arabe, la langue officielle du pays. Il s'agit donc d'un bilinguisme délibérément accepté où l'arabe serait à la fois la langue de l'identité arabo-musulmane et celle de l'accès au développement et le français, résolument conservé, servirait d'outil de travail privilégié dans la vie économique et financière.

3 Pratiquée par une bonne partie de la population locale principalement dans les villes et dans les milieux instruits, la langue française continue de servir d'outil de travail immanquable dans la vie économique et financière. Elle continue également, en dépit de la Mondialisation et de l'anglicisme massif qu'elle impose, d'être la langue étrangère la plus privilégiée par les opérateurs économiques nationaux.

4 Au sein des PME marocaines, la langue française jouit, à côté de l'arabe, d'une importance capitale. Elle est utilisée à des degrés divers, par les dirigeants et les collaborateurs dans leurs communications écrites (lettre, note de service, rapport, compte rendu...), orales 
(téléphone, réunion, entretien...) et médiatisées (Intranet, Extranet, messagerie électronique...).

\section{Problématique générale}

5 Quel type de PME marocaines fait-il appel à l'usage de la langue française ? Et à quelle fréquence?

6 A quelles images et à quels attributs la langue française est-elle associée dans l'esprit des entrepreneurs marocains?

7 Y a-t-il acceptation ou rejet de cette langue étrangère par les collaborateurs exerçant dans des PME marocaines? Et en raison de quelles motivations?

8 C'est autour de ces questions que s'articule notre problématique générale. L'ultime objectif à atteindre serait de jeter un regard analytique sur les conceptions intériorisées ainsi que sur les pratiques effectives liées à l'usage de la langue française en milieu professionnel marocain.

9 Pour ce faire, nous allons à la fois présenter et interpréter les résultats obtenus à partir d'une enquête que nous avons conduite sur le terrain en 2007. Il s'agit de deux questionnaires destinés respectivement aux dirigeants des PME et aux collaborateurs relevant de trois régions du Royaume, à savoir : Casablanca, Marrakech et Béni Mellal.

\section{Enquête}

10 L'enquête a été menée en deux temps différents : d'abord une enquête qualitative dont l'outil d'investigation était l'entretien semi-directif, ensuite une enquête quantitative dont l'instrument de mesure était le questionnaire. Les indicateurs subjectifs tirés à partir de l'entretien nous ont largement aidés à la conception des items contenus dans le questionnaire.

11 Le questionnaire destiné aux dirigeants a été distribué à 74 sujets relevant de trois grandes régions du Royaume, à savoir: Casablanca, Marrakech et Béni Mellal. De 74 exemplaires distribués, nous avons pu récupérer 50 , soit $67.56 \%$. Quant à celui destiné aux collaborateurs, il a été distribué à 250 sujets exerçant au sein des PME. De 250 exemplaires distribués, nous avons pu récupérer 145 (soit 58 \%) dûment remplis.

12 L'échantillon expérimental a été choisi au hasard selon les principes de base de l'échantillonnage aléatoire. Certaines variables se rapportant à l'âge, au sexe et à l'ancienneté ont été donc contrôlées au cours de ce choix.

\section{Présentation et discussion des résultats}

\section{Questionnaire destiné aux dirigeants des PME}

13 Item 1 : D'après vous, à quoi l'usage du français au sein de l'entreprise est-il associé ?

- A un facteur de réussite

- A un facteur de prestige

- A un facteur d'échec

- A un facteur d'aliénation 
Figure 1 : L'image du français au sein de l'entreprise

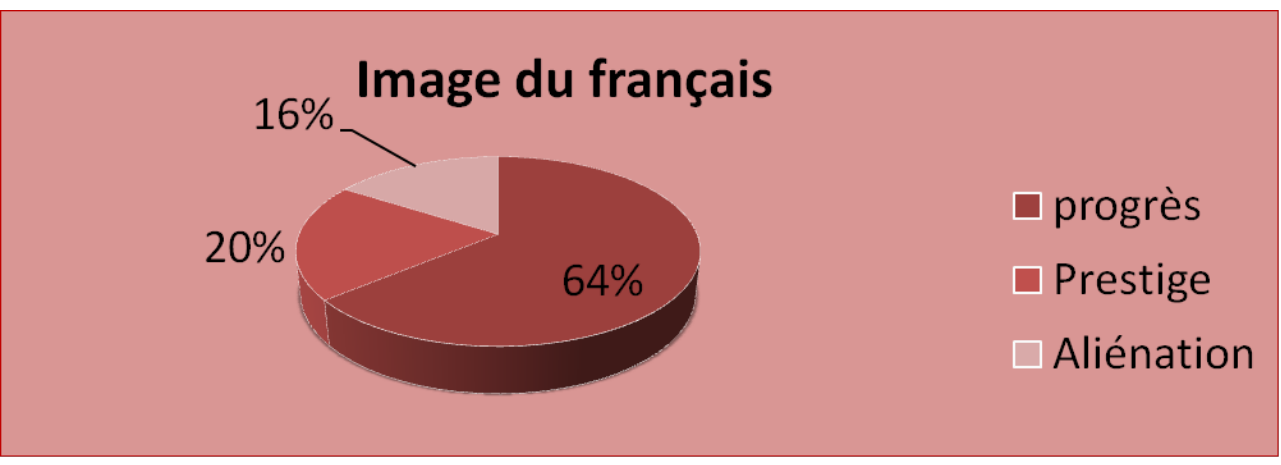

14 Item 2 : La maîtrise de la langue française est pour vous :

- Un moyen nécessaire pour être au courant du progrès

- Une ouverture sur le monde extérieur

- Un moyen nécessaire pour cultiver son imaginaire

- Un atout pour faire partie d'une élite sociale

- Un danger pour le mode de vie traditionnel

Figure 2 : Conceptions à propos de la maîtrise de la langue française

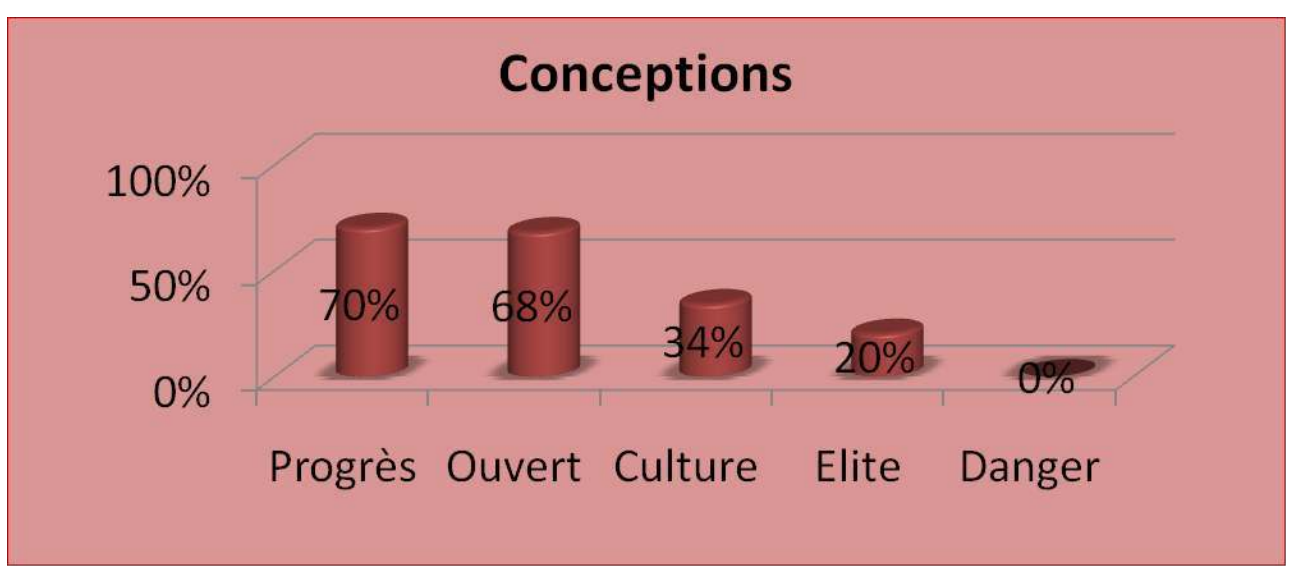

15 Item 3: Au sein de votre entreprise, le bilinguisme (arabe/français) constitue-t-il un facteur de richesse?

- Tout à fait

- Suffisamment

- Pas assez

- Pas du tout 
Figure 3 : Le bilinguisme (arabe/français) au sein de l'entreprise

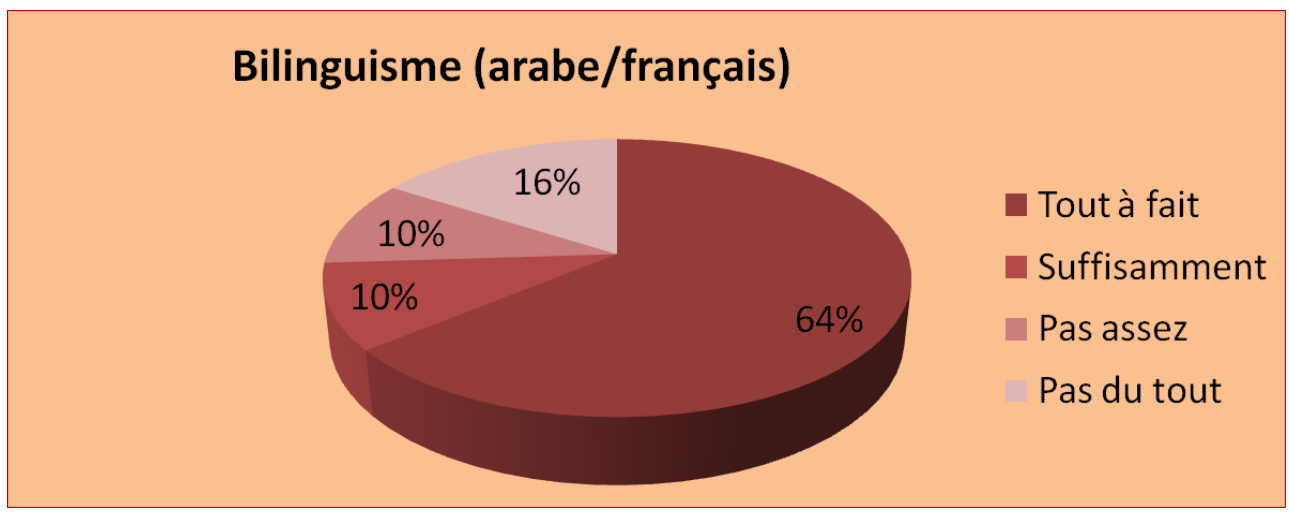

16 Item 4 : Quel est le pourcentage correspondant à l'usage du français dans la production de vos communications écrites, orales et médiatisées?

Tableau 1 : Usage du français dans la production des communications écrites, orales et médiatisées

\begin{tabular}{|l|l|l|l|}
\hline Communication & Ecrite & Orale & Médiatisée \\
\hline$(0 \%, 25 \%)$ & $0 \%$ & $12 \%$ & $4 \%$ \\
\hline$(26 \%, 50 \%)$ & $4 \%$ & $24 \%$ & $8 \%$ \\
\hline$(51 \%, 75 \%)$ & $14 \%$ & $34 \%$ & $6 \%$ \\
\hline$(76 \%, 100 \%)$ & $82 \%$ & $30 \%$ & $60 \%$ \\
\hline Pas de réponse & $0 \%$ & $0 \%$ & $22 \%$ \\
\hline Total & $100 \%$ & $100 \%$ & $100 \%$ \\
\hline
\end{tabular}

17 Item 5: Quel type de français utilisez-vous dans vos activités professionnelles? Et à quelles fréquences? 
Figure 4 : Usage du français général et du français de spécialité dans l'entreprise

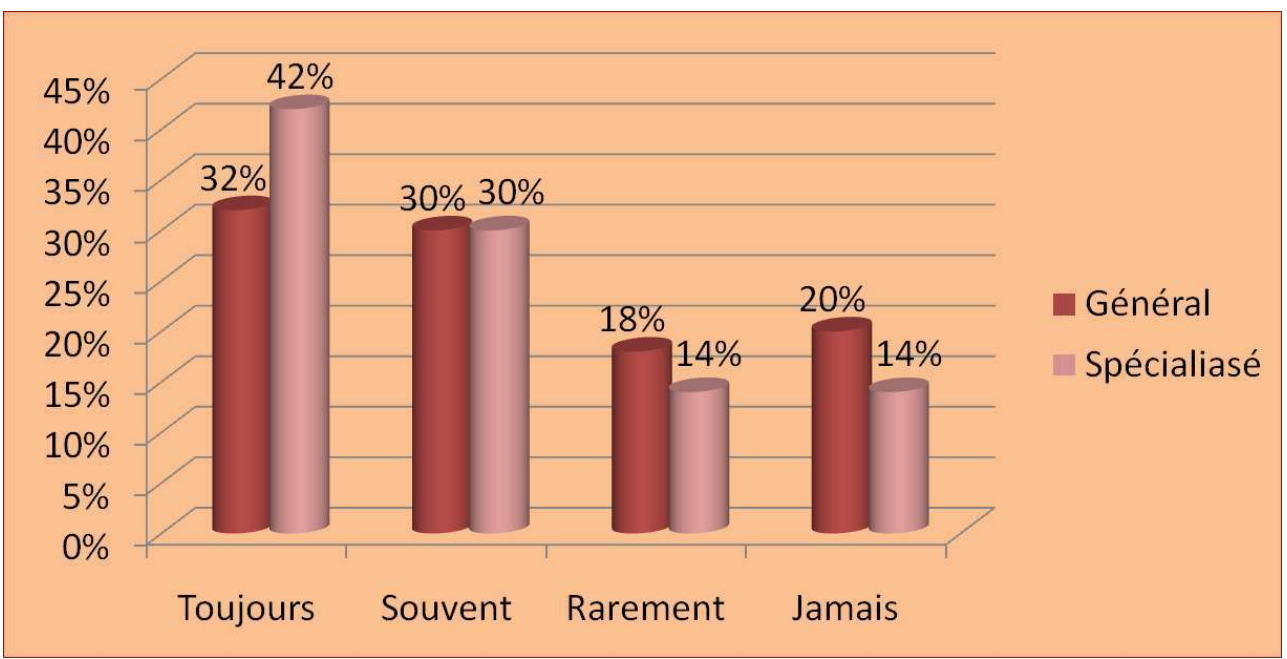

Item 6: A quelle fréquence, utilisez-vous le français pour communiquer avec vos collaborateurs?

- Toujours

- Souvent

- Rarement

- Jamais

Figure 5 : Usage du français dans la communication avec les collaborateurs (ici)

\section{Communication avec les collaborateurs}

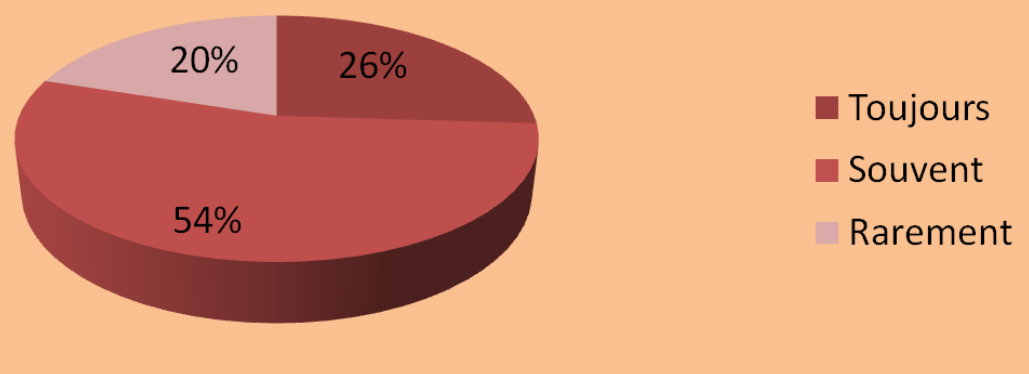

19 Item 7: Est-ce que le niveau de maîtrise du français figure parmi les critères de recrutement auxquels vous donnez plus d'importance?

- Toujours

- Souvent

- Rarement

- Jamais 
Figure 6 : Importance du niveau de maîtrise du français dans le recrutement des candidats

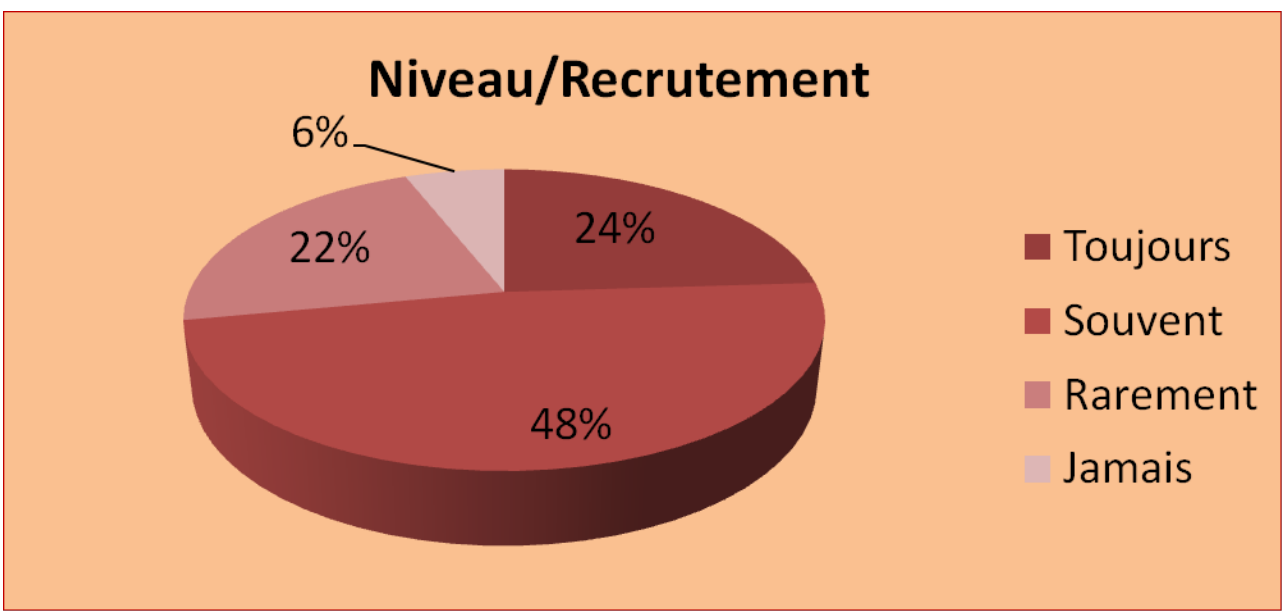

Item 8 : Au sein de votre entreprise, quel type de compétence se rapportant à l'usage de la langue française cherchez-vous à développer?

- Une compétence linguistique

- Une compétence communicative

- Une compétence socioculturelle

- Une compétence stratégique

Figure 7 : Types de compétence en rapport avec la langue française

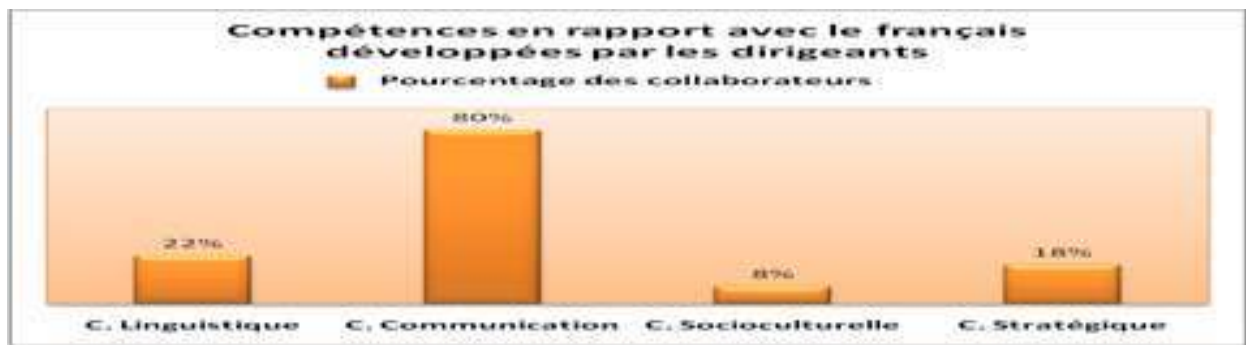

Item 9 : D'après vous, la langue française, en tant qu'outil de travail privilégié, peut-elle permettre à l'entreprise de développer une stratégie de communication?

- Oui

- Non

- Pourquoi, justifiez votre réponse 
Figure 8 : Rôle du français dans le développement d'une stratégie de communication

\section{Français/Stratégie de communication}

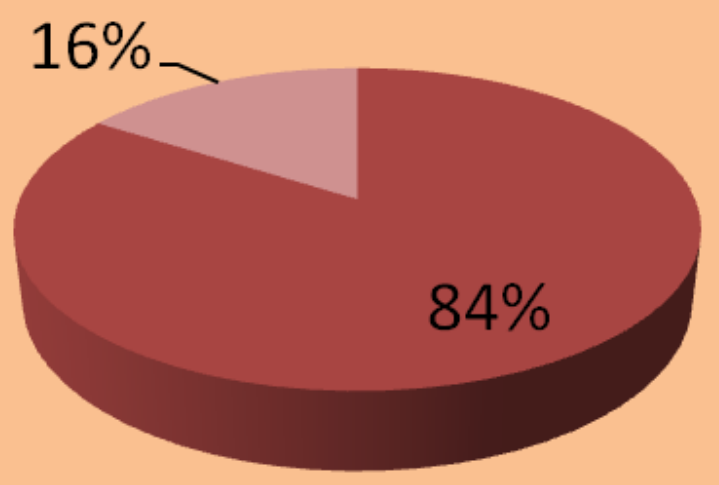

Figure 9 : Justifications (français/stratégie de communication)

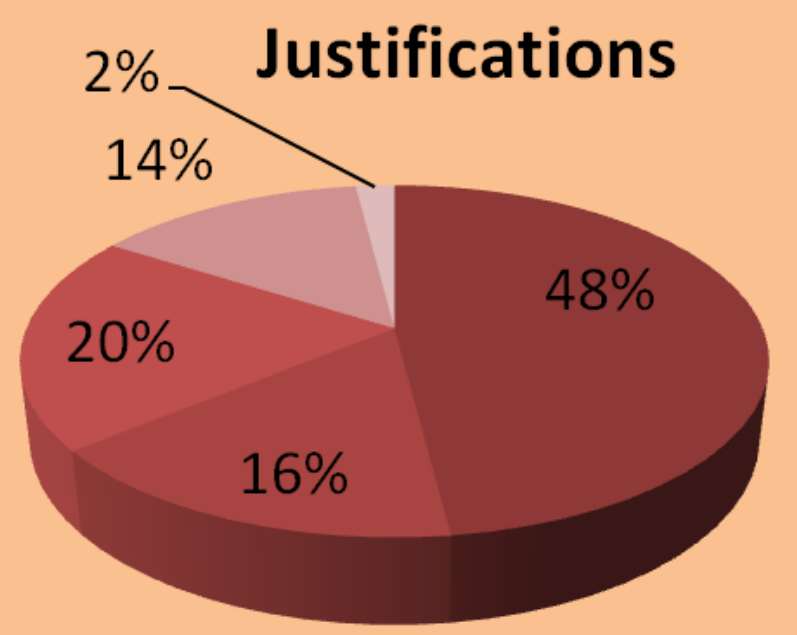

1- Pas de réponse

2- Oui, si la clientèle est à la fois scolarisée et francisée

3- La communication passe nécessairement par la langue française

4- Le français est un outil de travail et un instrument au service de la Mondialisation

5- Non, le produit prime

Item 10 : L'usage fonctionnel de la langue française peut-il amener l'entreprise à réaliser une meilleure rentabilité et une hausse de son chiffre d'affaires?

- Oui

- Non

- Pourquoi, justifiez votre réponse 


\section{Français/Chiffre d'affaires}

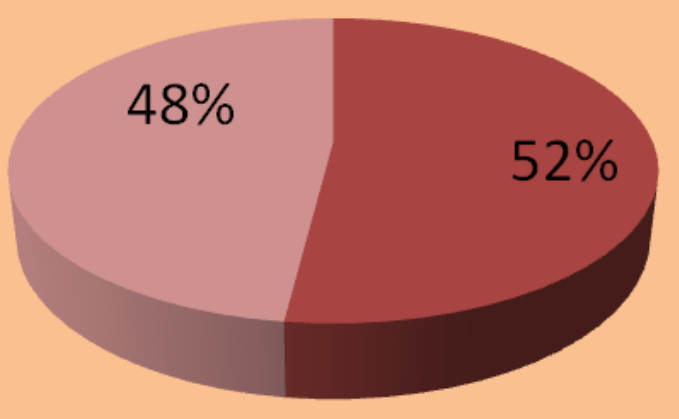

Oui

Non

Figure 11 : Justifications (français/chiffre d'affaires)

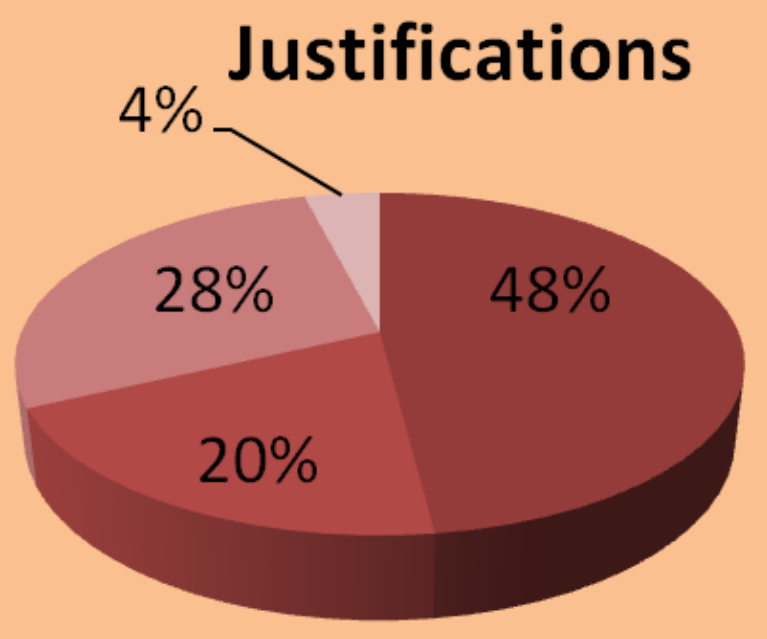

1- Pas de réponse

2- D'autres facteurs entrent en jeu comme la gestion, l'emplacement, les tarifs, la qualité du service...

3- L'usage du français est rentable avec les clients francisés, c'est un atout susceptible d'attirer davantage la clientèle

4- Dans les petites villes, l'usage du français est rarement sollicité

Item 11 : Comment concevriez-vous l'entreprise marocaine?

- Avec une suprématie de l'arabe

- Avec une suprématie du français

- Les deux sur le même niveau

- Avec l'arabe seulement

- Pourquoi, justifiez votre réponse 
Figure 12 : Conception future des dirigeants à propos de l'aménagement linguistique au sein de l'entreprise marocaine

\section{Conception future}

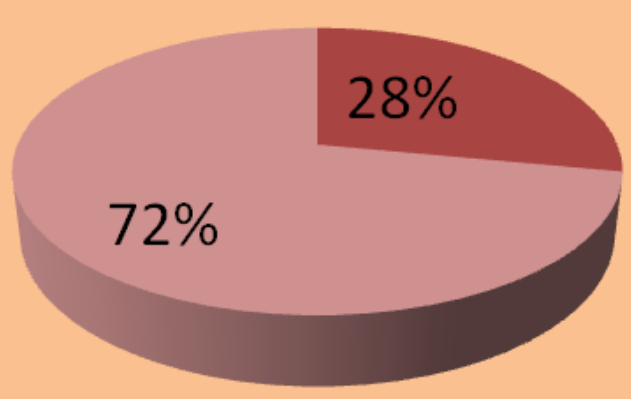

Suprém fr

Arab+fr

Figure 13 : Justifications (conception des dirigeants : arabe/français)

\section{Justifications}

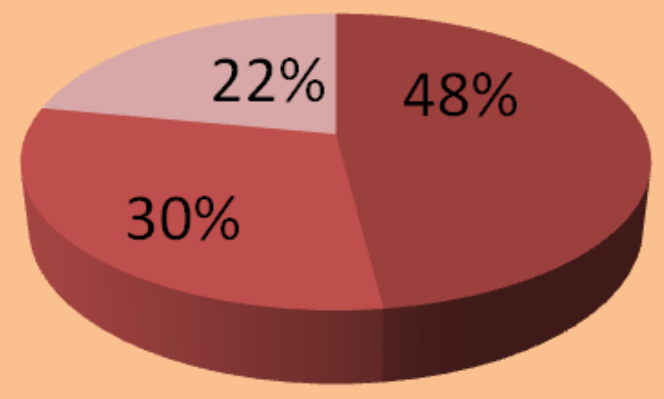

1- Pas de réponse

2- L'arabe constitue la langue de notre identité arabo-musulmane alors que le français reste un outil de travail privilégié

3- Le français n'est pas nécessaire à l'entreprise de demain, c'est l'anglais qui prévaut

\section{Interprétation des résultats}

\section{1/ Images associées à l'usage de la langue française au sein de l'entreprise}

De l'observation de la figure 1, il ressort qu'aucun des sujets enquêtés ne lie l'usage de la langue française au sein de l'entreprise à un facteur d'échec. Bien au contraire, la majorité (soit $64 \%$ ) trouve que c'est un facteur de réussite qui permet à l'entreprise d'aller de l'avant.

$20 \%$ estiment que c'est un facteur de prestige qui dote celui qui la pratique d'une certaine prééminence. Dans ce raisonnement, il est sous-entendu que celui qui utilise le français 
dans son travail est supérieur par rapport à celui qui utilise l'arabe. La langue serait-elle donc un outil discriminatoire?

Ceci dit, l'écart entre ceux qui considèrent l'usage du français au sein de l'entreprise comme un facteur de réussite (soit $64 \%$ ) et ceux qui estiment que c'est un facteur d'aliénation (soit $16 \%$ ) plaide sans conteste au profit d'une nette valorisation de la langue française dans le monde des affaires.

Parallèlement à la figure 1, la série des pourcentages représentés dans la figure 2, montre de toute évidence que la totalité des sujets enquêtés (soit 50 sujets sur un total de 50) pense que la maîtrise de la langue française ne constitue pas un danger pour le mode de vie traditionnel. De là, la langue française a toute légitimité d'existence dans une société telle que la nôtre, et ce sans aucune rivalité avec la langue arabe.

Une légitimité d'existence qui prédispose la langue française de qualités superlatives ayant trait à la modernité, au progrès et à l'ouverture. Ainsi, pour $70 \%$ de nos sujets, la maîtrise $d u$ français est un moyen nécessaire pour être au courant du progrès. Dans l'imaginaire collectif de nos dirigeants, le français reste donc une langue de diffusion du savoir scientifique et technologique par excellence.

L'ouverture sur le monde extérieur vient en seconde position avec un pourcentage de $68 \%$. C'est l'appel à l'ouverture, l'accès aux marchés extérieurs surtout francophones, le désir d'explorer d'autres horizons : un trait caractéristique de la personnalité maghrébine qui demeure tolérante, ouverte et prête à apprendre les langues étrangères.

Un moyen nécessaire pour cultiver son imaginaire vient en troisième position avec un pourcentage de $34 \%$. En plus d'être une langue de progrès et d'ouverture, le français serait une langue de culture qui permet à celui qui la pratique de développer un savoir cognitif étendu.

31 Une minorité de $20 \%$ voit que c'est un atout pour faire partie d'une élite sociale. La question de discrimination revient encore. Mais, vu les proportions négligeables que cela représente, nous pouvons dire que l'image d'aliénation ou de discrimination socioéconomique à laquelle renvoie l'usage du français intègre la série des préjugés que dégage l'usage d'une langue étrangère de grande diffusion comme le français dans une société en voie de développement comme la nôtre.

\section{2/ Bilinguisme (arabe/français) et importance accordée au français au sein de l'entreprise}

32 A la lecture de la figure 3, il semble que la majorité des dirigeants voient que le bilinguisme (arabe/français) constitue tout à fait (soit $64 \%$ ), sinon suffisamment (10\%) un facteur de richesse au sein de leurs entreprises. Partant de là, être bilingue est plus qu'un atout, c'est une nécessité qui s'impose avec acuité à tout porteur de projet.

Il s'agit donc d'un bilinguisme délibérément accepté où l'arabe faciliterait l'échange avec les institutions publiques ainsi que l'accès au marché national et le français serait un outil de travail privilégié ayant trait à la modernité, au progrès et à l'ouverture sur les marchés francophones.

Par ailleurs, l'analyse comparative des pourcentages de choix représentés dans le tableau 1 montre que la plupart des sujets utilisent le français (de $75 \%$ à $100 \%$ ) dans leurs productions écrites (soit $82 \%$ ) et médiatisées (soit $60 \%$ ). 
35 La communication orale en français revêt également de l'importance dans les pratiques quotidiennes des dirigeants mais elle est utilisée diversement. Enfin, il reste à signaler que $22 \%$ des sujets n'ont pas jugé utile de donner leurs avis sur l'usage du français en communication télématique. Ce refus signifie, soit qu'ils ne font pas encore usage de l'outil informatique et des NTIC, soit que le terme « médiatisé » ou « télématique » reste à leurs yeux méconnu, voire confus.

\section{3/ Usage du français général et du français de spécialité dans l'entreprise}

36 A la lecture de la figure 4, nous constatons que le français général et le français de spécialité sont utilisés à presque de part égale par les dirigeants des PME avec une légère prééminence pour le français de spécialité. Ainsi, $42 \%$ de nos sujets recourent toujours à l'usage du français de spécialité, $30 \%$ l'utilisent souvent, $14 \%$ l'utilisent rarement et $14 \%$ ne l'utilisent jamais. Parallèlement, $34 \%$ des sujets font toujours usage du français général, $30 \%$ l'utilisent souvent, $18 \%$ l'utilisent rarement et $20 \%$ ne l'utilisent jamais.

37 A partir de ces proportions approximativement similaires, il paraît évident que les PME marocaines utilisent modérément à la fois le français littéraire et le français fonctionnel. Le premier trouve son champ d'application en communication orale et écrite. Le second, lui, s'utilise partout en communication écrite, orale et télématique.

Sachant que $74 \%$ des entreprises recensées opèrent dans le secteur tertiaire, le français commercial trouve son emploi dans les transactions orales. De même, en communication médiatisée, les logiciels sont généralement conçus en français spécialisé. Il s'agit au fait d'une sorte de sabir technique sur lequel les collaborateurs se sont largement entraînés.

Ceci dit, nous persistons tout de même à signaler que le français de spécialité n'est autre qu'un sous-système du français général et qu'il s'agit, bien entendu, de la même langue organisée selon les besoins spécifiques de chaque entreprise.

\section{4/ le français au sein de l'entreprise}

De l'observation de la figure 5, il parait qu'aucun des sujets enquêtés n'utilisent jamais le français pour communiquer avec les collaborateurs. La majorité des sujets (soit $54 \%$ ) avouent recourir souvent au français pour communiquer avec le personnel. Cette facilité de communication, fort impressionnante, relève du niveau d'instruction relativement élevé dont témoigne la majorité de nos dirigeants ( $78 \%$ d'entre eux ont suivi des études supérieures allant du DEUG au Doctorat).

Il reste juste à vérifier si le principe de feed-back est doublement assuré, car tout acte d'interaction suppose un aller-retour de l'information. Ceci nous pousse à nous poser des questions sur le degré de maîtrise du français chez l'ensemble des collaborateurs.

Par ailleurs, la maîtrise du français reste l'un des critères les plus requis dans le recrutement d'un candidat. D'ailleurs, en consultant les pourcentages représentés dans la figure 6, nous apprenons que $24 \%$ des dirigeants font toujours attention au niveau de maîtrise du français du candidat avant de l'engager, $48 \%$ y prêtent souvent attention, $22 \%$ y prêtent rarement attention et seulement $6 \%$ n'y prêtent jamais attention. Plus qu'un atout, la maitrise des langues étrangères devient donc une nécessité pour tout demandeur d'emploi car dans un entretien d'embauche, les connaissances techniques et la pratique des langues étrangères prennent le pas sur la maitrise de la langue maternelle. 


\section{5/ Rôles et fonctions de la langue française au sein de l'entreprise}

D'après la figure 7, la majorité écrasante des dirigeants (soit $80 \%$ ) aspirent à développer à travers l'usage de la langue française une compétence communicative. Ceux qui cherchent à développer une compétence linguistique représentent un pourcentage de $22 \%$.

44 Autrement dit, la priorité est donnée au discours sur la langue, à l'usage sur le système et à la performance sur la compétence. Ceci dit, il serait difficile, voire impossible de dissocier la langue de la communication. La qualité de la syntaxe et la richesse du lexique font partie des contenus dont la communication a besoin pour être opérationnelle.

45 Partant de là, la compétence linguistique fait partie de la compétence communication au même titre qu'il existe une compétence stratégique et une compétence socioculturelle. Ainsi, $18 \%$ de notre échantillon entendent développer une compétence stratégique en rapport avec l'usage du français et $8 \%$ entendent développer une compétence socioculturelle.

De l'observation des figures 8 et 9 , il semble que pour $84 \%$ des dirigeants, la langue française en tant qu'outil de travail privilégié, peut permettre à l'entreprise de développer une stratégie de communication. En conséquence, $20 \%$ d'entre eux estiment que ceci est possible avec une clientèle scolarisée et francisée, $16 \%$ voient que la communication passe nécessairement par le français, $14 \%$ font du français à la fois un instrument de travail et un outil au service de la Mondialisation.

Ces proportions bien que négligeables renseignent d'une part sur la prise de conscience des entrepreneurs marocains de la composante communicative que dégage l'usage du français au sein de leurs entreprises et d'autre part sur la nécessité de maitriser le français pour faire face au poids de la Mondialisation et faciliter l'accès aux marchés extérieurs.

Il ressort de tous ces résultats que la majorité des dirigeants aspirent à développer une compétence communicative globalisante relative à l'usage du français au sein de leurs entreprises. Toutefois, nous rappelons que la majorité des dirigeants investissent peu en communication externe et interne.

Entre la théorie et la pratique se creuse donc un grand fossé. Autrement dit, les chefs d'entreprises sont conscients de l'apport positif de la composante communicative dans l'amélioration de leurs productivités. Néanmoins, cette prise de conscience ne passe guère à l'action. Est-ce par manque de disponibilité ou l'investissement en communication se voit encore coûteux ? Il est notoire que les deux probabilités soient des explications admissibles, mais ce qu'il faut réellement retenir, c'est que le concept de communication reste encore un sujet «tabou» aussi bien dans l'esprit que dans les pratiques quotidiennes d'un bon nombre de micro-entrepreneurs marocains.

\section{6/ Contribution de la langue française dans la hausse du chiffre d'affaires}

De l'observation des pourcentages de choix représentés dans la figure 10, il apparaît que les sujets interrogés se partagent entre ceux qui perçoivent un impact positif de l'usage fonctionnel de la langue française sur la productivité de l'entreprise (soit $52 \%$ ) et ceux qui n'en voient aucun rapport (soit $48 \%$ ). Un tel résultat dont les fréquences sont à peine dispersées avec un léger avantage pour la première acception nous laisse réfléchir: 
l'usage du français peut-il vraiment permettre à l'entreprise de réaliser une hausse de son chiffre d'affaires?

En nous référant aux justifications des enquêtés exposées dans la figure 11, nous trouvons que pour $28 \%$ de notre échantillon, l'usage fonctionnel du français est rentable avec les clients francisés, c'est un atout susceptible d'attirer davantage la clientèle. Ce raisonnement est valable surtout dans les grandes villes où le taux de civilité et d'urbanité est assez élevé par rapport aux petites agglomérations.

$20 \%$ de notre échantillon pensent que la rentabilité de l'entreprise n'est nullement liée à une question de langue, elle reste cependant fonction de certains critères stratégiques comme : l'emplacement, les tarifs concurrentiels, la qualité du service, la bonne gestion... $48 \%$ des sujets restants se sont abstenus de donner leurs avis. Leur refus signifie soit un rejet de la question ouverte, soit qu'ils partagent l'argument de ceux pour qui la hausse du chiffre d'affaires dépend largement des facteurs stratégiques mentionnés ci-dessus.

\section{7/ Conception future des entrepreneurs à propos de l'aménagement linguistique au sein de l'entreprise marocaine}

53 En nous référant à la figure 12, notre surprise était grande de constater qu'aucun des sujets interrogés ne reconnaît la suprématie de l'arabe dans les années à venir au sein de l'entreprise marocaine. La majorité écrasante (soit $72 \%$ ) conçoit l'usage de l'arabe et du français sur le même niveau alors que $28 \%$ reconnaissent même une certaine prééminence du français par rapport à l'arabe.

De là, il ressort que l'éventualité d'un bilinguisme (arabe/français) est délibérément consentie par les entrepreneurs marocains. D'ailleurs, en nous référant à la figure 13, nous comprenons que pour $30 \%$ de notre échantillon, l'arabe constitue la langue de l'identité arabo-musulmane et le français représente un outil de travail immanquable.

Nous rappelons également que pour $22 \%$ de nos sujets, l'accent doit être mis plutôt sur la langue anglaise car elle incarne la langue de l'avenir et de la Mondialisation. Cette proportion sous-entend donc la prise de conscience d'un certain nombre de dirigeants marocains du changement que la Mondialisation et ses corollaires pourraient éventuellement apporter au niveau de l'aménagement linguistique du pays.

Des résultats aussi impressionnants soient-ils, nous laissent perplexe et nous poussent à nous poser cette question : comment expliquer le recours massif au français des affaires dans un pays arabo-musulman dont la langue officielle est l'arabe?

La langue arabe défavorisée signifie que la politique économique suivie aménage les espaces à investir par les langues étrangères avec une prévention qui ne favorise aucunement l'épanouissement de la langue officielle du pays. Autrement dit, la politique économique n'encourage pas la langue nationale, comme il se doit.

Faute de quoi, la langue française préservera sereinement son statut de leader dans le monde des affaires à l'échelle nationale. Un statut qui risque d'être concurrencé par l'anglais, devenu « lingua franca » du monde contemporain. 


\section{Questionnaire destiné aux collaborateurs exerçant au sein des PME marocaines}

Item 1 : Quelle conception avez-vous de la langue française?

- Langue de progrès

- Langue de modernisme

- Langue d'ouverture sur l'occident

- Langue d'une élite sociale

- Séquelle du colonialisme

- Langue rivale de la langue maternelle

- Autre (à préciser) : langue des affaires / langue de communication

Figure 1 : Conceptions des collaborateurs à propos de la langue française

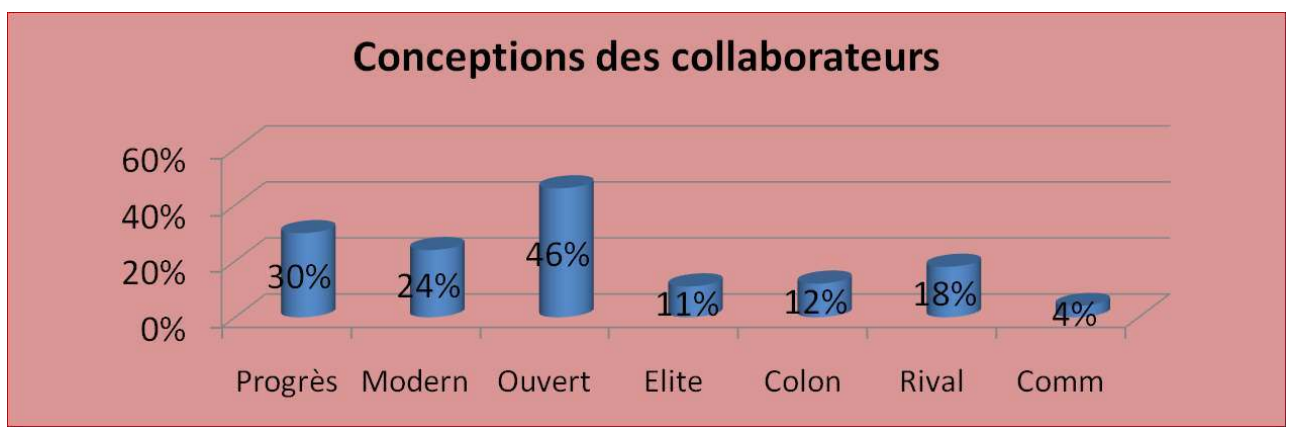

Item 2 : Quel est votre degré d'intériorisation du français?

- Acceptation

- Usage sans complexe

- Usage avec complexe

- Rejet

Figure 2 : Degré d'intériorisation de la langue française par les collaborateurs

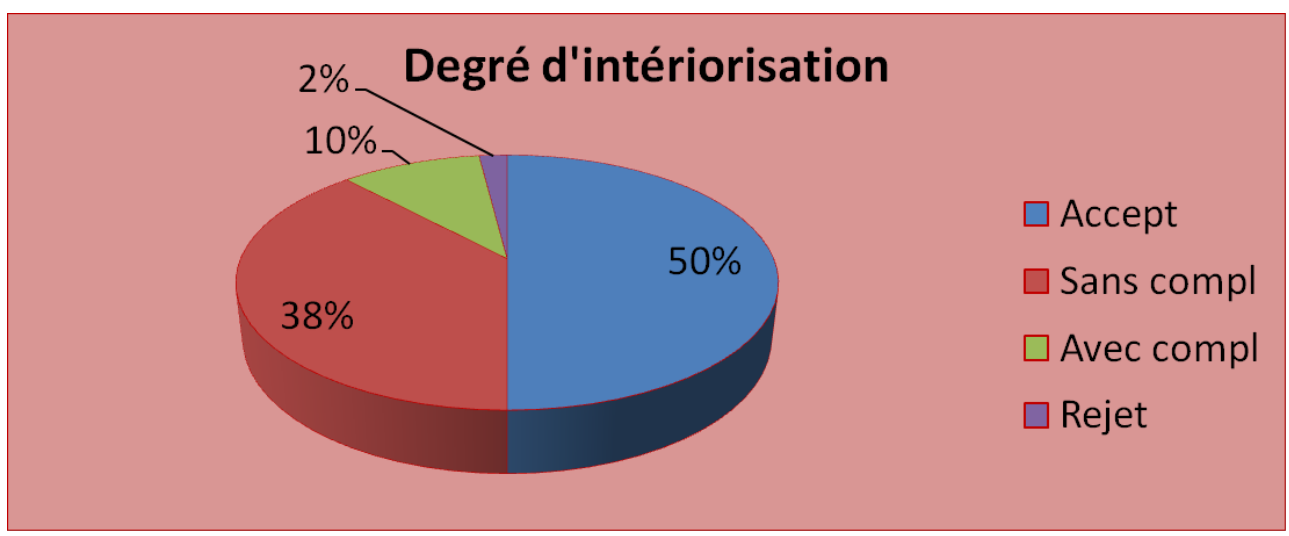

Item 3 : Compte tenu de votre expérience professionnelle, la langue française :

- Vous aide-t-elle à réussir?

- Est-ce la cause de votre échec?

- N'a aucun effet sur votre carrière

- Pas de réponse 
Figure 3 : Effet de la langue française sur la carrière des collaborateurs

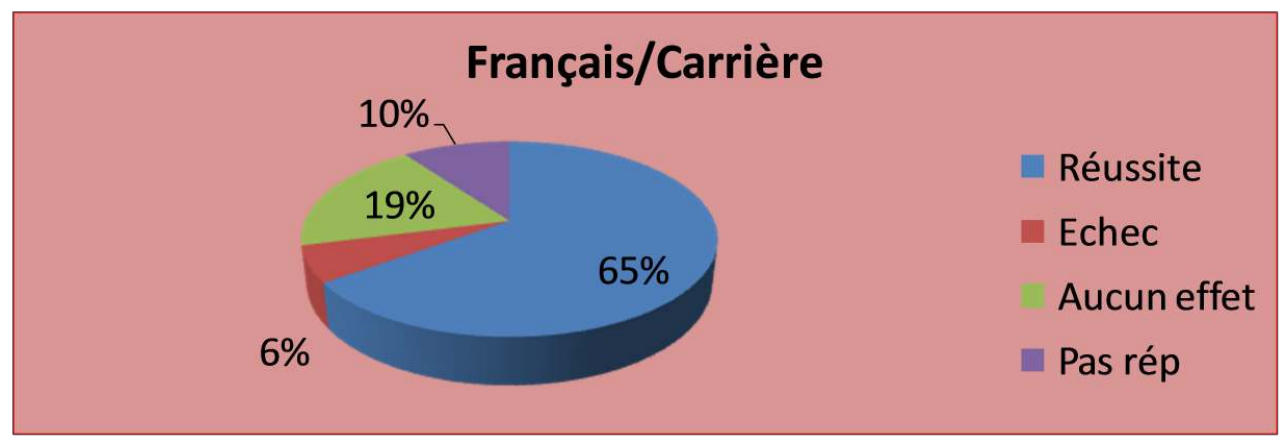

Item 4 : Quelle attitude adoptez-vous lorsqu'un collègue vous parle en langue française ?

- Etonnement

- Surprise agréable

- Admiration

- Gêne

- Mépris

- Attitude neutre

Figure 4 : Attitudes des collaborateurs face à un collègue s'exprimant en français

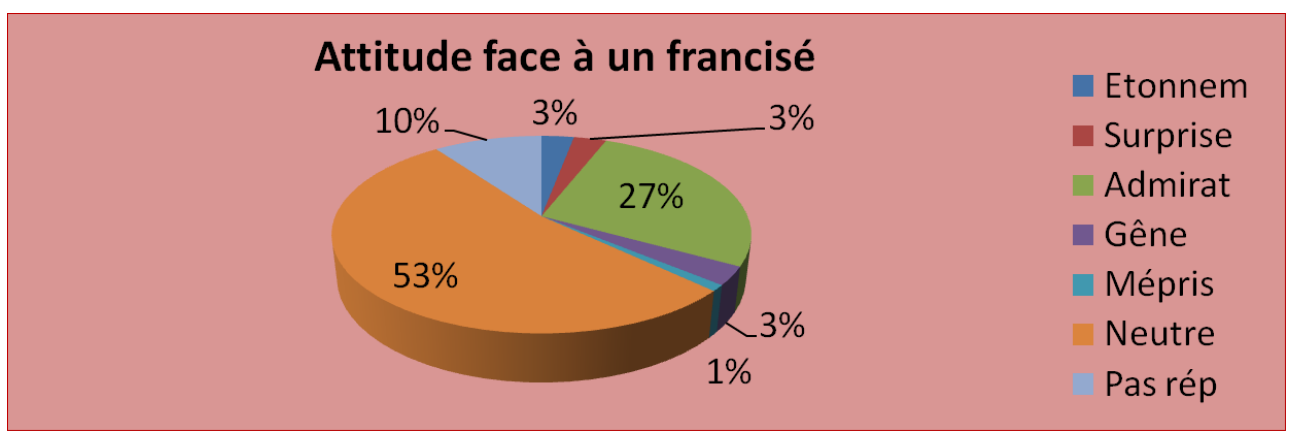

Item 5 : Quelle place le français occupe-t-il par rapport aux autres langues en présence?

- Très importante

- Importante

- Assez importante

- Peu importante

- Pas du tout importante 
Figure 5 : Place du français parmi les autres langues en présence

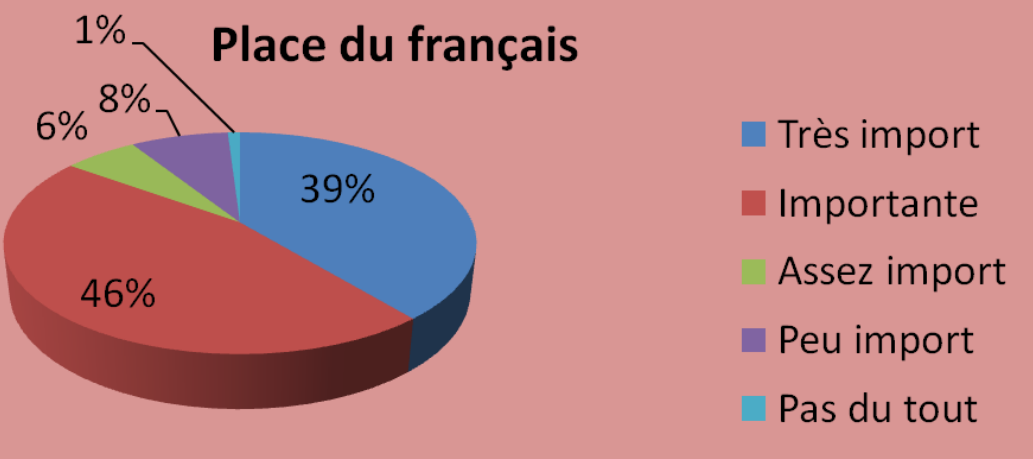

Item 6: Quel est le pourcentage correspondant à l'usage du français dans la production de vos communications?

Tableau 1 : Usage du français dans les communications des collaborateurs

\begin{tabular}{|l|l|l|l|l|l|l|}
\hline Communication & $(0 \%, 25 \%)$ & $(26 \%, 50 \%)$ & $(51 \%, 75 \%)$ & $(76 \%, 100 \%)$ & Pas réponse & Total \\
\hline Ecrite & $8 \%$ & $15 \%$ & $17 \%$ & $48 \%$ & $12 \%$ & $100 \%$ \\
\hline Orale & $13 \%$ & $20 \%$ & $42 \%$ & $18 \%$ & $7 \%$ & $100 \%$ \\
\hline Médiatisée & $14 \%$ & $17 \%$ & $15 \%$ & $26 \%$ & $28 \%$ & $100 \%$ \\
\hline
\end{tabular}

Item 7 : Les documents que l'on vous demande de rédiger en langue française vous semblent

- Faciles

- De votre niveau

- Difficiles

- Incompréhensibles

Tableau 2 : Difficultés des documents rédigés par les collaborateurs

\begin{tabular}{|l|l|l|l|l|l|l|}
\hline Niveau & Toujours & Souvent & Rarement & Jamais & Pas rép. & Total \\
\hline Faciles & $26 \%$ & $43 \%$ & $3 \%$ & $1 \%$ & $27 \%$ & $100 \%$ \\
\hline De votre niveau & $27 \%$ & $32 \%$ & $6 \%$ & $1 \%$ & $34 \%$ & $100 \%$ \\
\hline Difficiles & $2 \%$ & $6 \%$ & $37 \%$ & $9 \%$ & $46 \%$ & $100 \%$ \\
\hline Incompréhensibles & $1 \%$ & $1 \%$ & $18 \%$ & $26 \%$ & $54 \%$ & $100 \%$ \\
\hline
\end{tabular}

Item 8 : Comment évaluez-vous votre niveau écrit et oral en français ? 
Tableau 3 : Niveau écrit et oral des collaborateurs en français

\begin{tabular}{|l|l|l|l|l|l|l|l|}
\hline Niveau & Très bon & Bon & Assez bon & Pas bon & Pas du tout bon & Pas de réponse & Total \\
\hline Ecrit & $11 \%$ & $42 \%$ & $39 \%$ & $5 \%$ & $0 \%$ & $3 \%$ & $100 \%$ \\
\hline Oral & $11 \%$ & $36 \%$ & $42 \%$ & $3 \%$ & $1 \%$ & $7 \%$ & $100 \%$ \\
\hline
\end{tabular}

67 Item 9: Quelles précautions prenez-vous pour développer votre compétence linguistique en français? (Plusieurs réponses sont possibles)

- Lire des documents élaborés en français

- Rédiger des documents en français

- Utiliser le dictionnaire

- Participer à des sessions de formations

- Prendre la parole en public

- Utiliser l'Internet

- Correspondre avec des francophones

- Autre (à préciser) : visionner des films, écouter des émissions à la radio

Figure 6 : Précautions prises par les collaborateurs pour développer une compétence linguistique en français

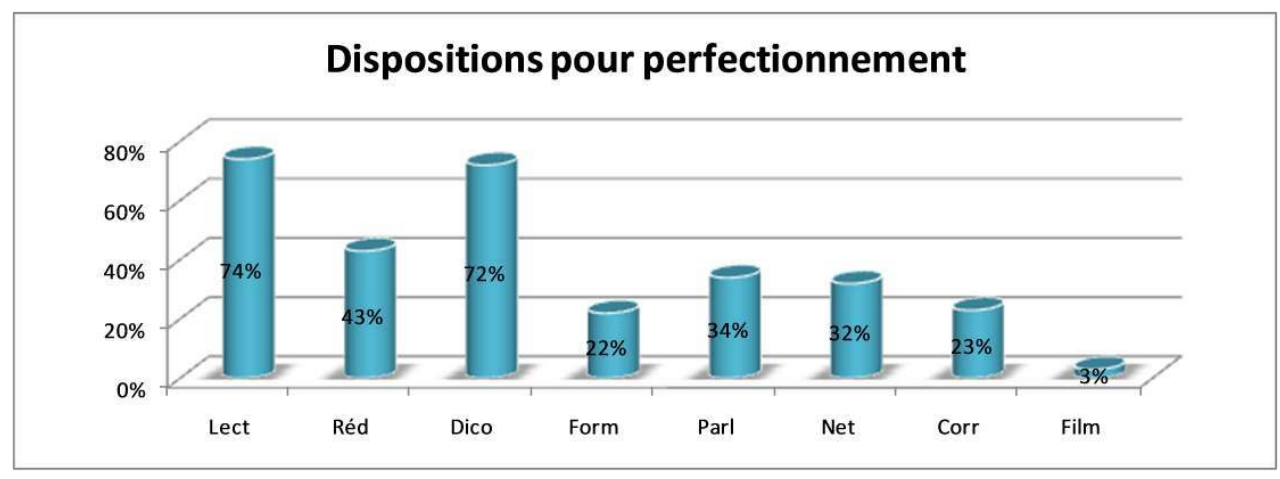

\section{Interprétation des résultats}

\section{1/ Images intériorisées chez les collaborateurs à propos de la langue française}

L'analyse comparative des pourcentages exposés dans la figure 1 montre que nos collaborateurs développent une conception plutôt positive à propos de la langue française. La majorité d'entre eux trouvent donc que c'est d'abord, une langue d'ouverture (soit $46 \%$ ), ensuite une langue de progrès scientifique et technologique (soit $30 \%$ ), enfin une langue moderne dotant celui qui la parle d'un certain prestige (soit $24 \%$ ).

En contre-partie, une autre minorité formée de $11 \%$ parle d'une langue qui taxe ceux qui la pratiquent d'appartenir à une élite sociale. La question de discrimination revient encore pour dénoter l'autorité qu'exerce une langue étrangère telle que la langue française dans un pays arabo-musulman tel que le nôtre. D'ailleurs, $18 \%$ de nos sujets estiment que le français représente une rivalité pour l'arabe, langue officielle du pays. En outre, les $12 \%$ restants le verbalisent clairement en considérant que le français comme 
une simple séquelle du colonialisme. Cette acception est de nature à nous faire comprendre que les tenants du discours traditionaliste continuent toujours à subsister.

Par ailleurs, en nous référant aux résultats représentés dans la figure 2, nous comprenons bien que la moitié de nos collaborateurs (soit $50 \%$ ) acceptent la langue française sans aucune réserve et $38 \%$ l'utilisent sans aucun complexe. Ces fréquences, aussi élevées soient-elles, témoignent de la conciliation presque parfaite des sujets avec la langue de Molière.

Ceci dit, $10 \%$ des sujets avouent l'utiliser avec un certain complexe et $2 \%$ la rejettent totalement. Ces dernières fréquences comparées aux deux premières restent insignifiantes mais il faut noter que de telles attitudes d'embarras ou de rejet signifient soit que les sujets sont monolingues, c'est-à-dire ils ne comprennent que l'arabe, soit que leur fanatisme à l'arabe cache une certaine hostilité vis-à-vis du français, l'ancienne langue du colonisateur.

Une part de vérité de ce qui vient d'être dit se confirme dans les résultats exposés dans la figure 3. En effet, $6 \%$ des collaborateurs pensent que la langue française est la cause principale de leur échec professionnel. Il existe donc un rapport de dépendance entre la maîtrise du français et la réussite professionnelle. Autrement dit, la maîtrise du français prédispose le salarié à développer des aptitudes intellectuelles susceptibles de le conduire à prospérer et à faire carrière. Une déduction qui s'applique à la majorité écrasante de nos sujets (soit $65 \%$ ) pour lesquels la langue française demeure l'un des secrets de leur réussite. L'écart entre les deux pourcentages plaide donc pour une nette valorisation de la langue française.

Cela malgré la proportion non négligeable des sujets ayant opté soit pour l'absence de rapport entre la maîtrise du français et la carrière professionnelle (19\%), soit pour l'abstention (10\%). Pour ces derniers, il est probable que la langue, qu'elle soit arabe, française ou anglaise, ne peut constituer un facteur de réussite ou d'échec et que ce sont les qualifications professionnelles et les ambitions qui permettent au salarié de promouvoir ou non.

Par ailleurs, à la lecture des résultats exposés dans la figure 4, nous constatons qu'un groupe de sujets déclare éprouver de l'admiration (soit $27 \%$ ) et une surprise agréable (3\% ) lorsqu'un collègue leur parle en français. Un autre groupe par contre avoue ressentir une certaine gêne (soit $3 \%$ ), de l'étonnement (3\%) et carrément du mépris ( $1 \%)$. Or, l'écart entre les pourcentages obtenus plaide sans aucune équivoque pour une mise en relief de la langue française.

En outre, plus de la moitié des sujets (soit $53 \%$ ) adoptent une attitude de neutralité face à un collègue s'exprimant en français. $10 \%$ des sujets se sont abstenus de donner leurs avis, mais nous pouvons comprendre qu'ils partagent l'avis de ceux qui adoptent une attitude de neutralité.

\section{2/ Importance accordée à la langue française}

De l'observation des pourcentages de choix exposés dans la figure 5 , nous nous rendons compte que les collaborateurs se font une représentation nettement utilitaire de la langue française dans l'exercice de leurs missions. Ainsi, la majorité des sujets précise que la langue française occupe par rapport aux autres langues en présence une place ou très importante (soit $39 \%$ ) ou importante (soit $46 \%$ ). De là, le coefficient de valeur accordé à 
la langue de Molière la dote d'un rôle instrumental et fait d'elle un outil de travail privilégié.

Cette importance, si elle est profondément ancrée dans l'esprit collectif de nos collaborateurs, elle l'est moins dans leurs pratiques quotidiennes. En effet, l'analyse comparative des pourcentages de choix représentés dans le tableau 1 montre bien que l'usage du français est peu recommandé dans les productions des sujets. A l'exception d'environ la moitié (soit $48 \%$ ) des sujets qui recourent (de $76 \%$ à $100 \%$ ) au français pour produire des documents de communication écrite, les autres formes de communications véhiculées en français sont relativement réduites. Autrement dit, soit les fréquences sont faibles, soit les sujets ne mentionnent pas leurs avis.

\section{3/ Difficultés des collaborateurs à utiliser le français}

78 L'embarras des collaborateurs se ressent surtout au niveau de leur obstination à reconnaitre les difficultés linguistiques auxquelles ils se trouvent confrontés lorsqu'on leur demande de rédiger des documents élaborés en français. A la lecture du tableau 2, il semble que les plus grands pourcentages figurent dans la colonne « pas de réponse ». De plus, une toute petite minorité $(1 \%$ ou $2 \%)$ reconnaît toujours la difficulté, sinon l'incompréhension des supports écrits que l'on exige d'eux. Autrement dit, la plupart des sujets trouvent que lesdits supports sont soit faciles, soit de leur niveau.

79 Si nous nous fions aux avis partagés, nous comprendrons bien que la maitrise du français ne constitue pas pour autant un souci majeur chez la plupart des sujets. D'ailleurs, en nous référant au tableau 3, nous nous rendons compte que $42 \%$ des sujets jugent bon leur niveau écrit en français et 39 \% le jugent assez bon. De même, 36 \% des sujets jugent bon leur niveau oral en français et $42 \%$ le jugent assez bon. Il existe certes un léger écart entre les différentes fréquences, mais il paraît clair que la majorité écrasante des sujets se vantent de maîtriser correctement le français tant sur le plan écrit que sur le plan oral. Un trait caractéristique des citoyens du grand Maghreb, la région qui abrite l'une des plus grandes communautés francophones au monde.

Or, il ressort de la figure 6 qu'un grand nombre de collaborateurs s'efforcent à prendre différentes précautions en vue de développer une compétence linguistique en français. Par compétence linguistique, nous entendons la maîtrise des règles linguistiques qui permettent à un interlocuteur de générer des énoncés sémantiquement et grammaticalement corrects. En d'autres termes, la compétence linguistique désigne l'aptitude d'une personne à comprendre, lire, écrire et parler une langue donnée.

81 Ainsi, $74 \%$ déclarent lire des documents élaborés en français spécialement les journaux et les revues ; $72 \%$ déclarent utiliser le dictionnaire pour vérifier l'orthographe, le sens et la transcription phonétique d'un mot; $43 \%$ s'entrainent à rédiger des documents en français surtout les lettres et les journaux intimes; 34 \% s'entraînent à s'exprimer oralement en français que ce soit en famille ou avec les amis ; $32 \%$ reconnaissent utiliser l'Internet pour faire des recherches et entrer en dialogue direct avec des francophones ; $23 \%$ avouent correspondre avec des francisés soit par e-mail, soit par courrier postal ; $22 \%$ avouent suivre des formations le soir pour perfectionner leur niveau en français et seulement $4 \%$ mentionnent l'intérêt des chaînes télévisées et des stations radio dans la diffusion des émissions et des films véhiculés en français. 

soient chefs d'entreprises ou travailleurs à l'égard de la langue française est tributaire des représentations mentales qu'ils se font de cette langue étrangère par rapport aux autres langues en présence. Dans la conjoncture actuelle, le français jouit d'une importance capitale car il constitue un outil de travail privilégié Seulement, il ne conduit ni à la hausse du chiffre d'affaires, ni au développement d'une stratégie de communication au sein de l'entreprise marocaine. D'ailleurs, l'investissement en communication et en gestion des ressources humaines est mis de côté par l'ensemble des micro-entrepreneurs marocains. Et ce, en raison de la faiblesse des capitaux et du manque de disponibilité des dirigeants ; l'accent est mis plutôt sur la production et la commercialisation du produit. professionnel national, cette même langue risque de céder la place à un anglais puissant et envahisseur dans les prochaines années à venir. L'avènement de la Mondialisation, la signature des accords de libre-échange que le Maroc a conclu récemment avec plusieurs pays anglophones, et le rapprochement entre le Nord et le Sud, sont autant des facteurs à même de croire à un changement s'opérer dans l'aménagement linguistique du pays où l'anglais deviendrait éventuellement la langue des affaires par excellence. Les chances de survie de l'arabe et du français, à l'échelle nationale, résideraient peut-être dans la chance de voir se développer un trilinguisme (anglais/arabe/français) que tout entrepreneur doit nécessairement maitriser pour commercialiser ses produits tant sur les marchés locaux que sur les marchés étrangers.

\section{BIBLIOGRAPHIE}

BENZAKOUR, F., D. GAADI, D. et QUEFFELEC, A. (2001). Le Français au Maroc. Lexique et contacts de langues. Louvain-la-Neuve : De Boeck-Duculot-Aupelf.

CARRE, P. (1991). Organiser l'apprentissage des langues étrangères : La formation linguistique professionnelle. Paris : Les éditions d'organisation.

DAKHLIA, J. (2004). Trames de langues : usages et métissages linguistiques dans l'histoire du Maghreb. Paris : Maisonneuve \& Larose.

Carnets, Deuxième série - 8 | 2016 
EL GHERBI, E. (1993). Aménagement linguistique et enseignement du français au Maroc : enjeux culturels, linguistiques et didactiques. Meknès : La Voix de Meknès.

MAJDI, T. (2010). « Place de la langue française dans les PME marocaines : conceptions intériorisées et images associées à son usage ", Revue Ivoirienne de Langues Etrangères (RILE), $\mathrm{N}^{\circ} 2$, cf. (http://www.rile-ci.org/articles/article2/Rile\%2010.pdf).

ROLAND, D. (2000). «FLE ou FLS : Le grand écart », Le français dans le monde, $\mathrm{n}^{\circ} 311$, juillet-août 2000, pp. 34-38.

\section{RÉSUMÉS}

$\mathrm{Au}$ sein des PME marocaines, la langue française jouit, à côté de l'arabe, d'une importance capitale. Elle est utilisée à des degrés divers, par les dirigeants et les collaborateurs dans leurs communications écrites (lettre, note de service, rapport, compte rendu...), orales (téléphone, réunion, entretien...) et médiatisées (Intranet, Extranet, messagerie électronique...).

L'objectif de cette recherche serait de jeter un regard analytique sur les conceptions intériorisées ainsi que sur les pratiques effectives liées à l'usage de la langue française en milieu professionnel marocain.

Pour ce, nous allons à la fois présenter et interpréter les résultats obtenus à partir d'une enquête que nous avons menée sur le terrain en 2006/2007. Il s'agit de deux questionnaires destinés respectivement aux dirigeants des PME et aux collaborateurs relevant de trois régions du Royaume, à savoir : Casablanca,

Within Moroccan SMEs, the French language has, alongside Arabic, of paramount importance. It is used to varying degrees by the executives and employees in their written communication (letter, memo, report, reporting ...), oral (telephone, meeting, interview ...) and telematic (Intranet, Extranet, e-mail ...).

The objective of this research would be to cast an analytical look at internalized conceptions as well as the actual practices related to the use of the French language in Morocco workplace.

For this, we will both present and interpret the results from a survey we conducted in the field in 2006/2007. There are two questionnaires respectively to SME managers and employees within three regions of the Kingdom, namely: Casablanca, Marrakech and Beni Mellal.

\section{INDEX}

Mots-clés : langue française, PME marocaines, usage, image, conception

Keywords : French language, Moroccan SMEs, the use, the image, the consideration

\section{AUTEUR}

\section{TOUFIK MAJDI}

Faculté Pluridisciplinaire de Khouribga

Université Hassan 1er Settat, Maroc

majditoufik[at]yahoo.fr 